EOMmUn: Communication et organisation

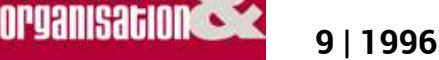

La communication des institutions religieuses

\title{
Les médias ont imposé une nouvelle logique à la religion
}

Bernard Dagenais

\section{(2) OpenEdition}

1 Journals

Édition électronique

URL : http://journals.openedition.org/communicationorganisation/1840

DOI : 10.4000/communicationorganisation. 1840

ISSN : $1775-3546$

Éditeur

Presses universitaires de Bordeaux

Édition imprimée

Date de publication : 1 mai 1996

ISSN : 1168-5549

Référence électronique

Bernard Dagenais, «Les médias ont imposé une nouvelle logique à la religion », Communication et organisation [En ligne], 9 | 1996, mis en ligne le 26 mars 2012, consulté le 19 avril 2019. URL : http:// journals.openedition.org/communicationorganisation/1840 ; DOI : 10.4000/

communicationorganisation. 1840

Ce document a été généré automatiquement le 19 avril 2019

(c) Presses universitaires de Bordeaux 


\title{
Les médias ont imposé une nouvelle logique à la religion
}

\author{
Bernard Dagenais
}

\section{NOTE DE L'AUTEUR}

Ce texte a tait l'objet d'une communication présentée le 27 juin 1995 par l'auteur à l'atelier « Religion et médias » de la XXIII ${ }^{e}$ Conférence de la Société Internationale de sociologie des religions, tenue à l'Université Laval (Québec, Canada) du 26 au 30 juin 1995.

1 Les médias ont envahi le monde de la politique, de la culture et de l'économie. A cause de leur omniprésence et de leur efficacité à « créer » des réalités, les médias ont Prescrit aux différents acteurs qui voulaient participer à la définition des enjeux sociaux, une logique de la "réalité ». La course à la médiatisation, par exemple, a poussé les organisations à reformuler leur présence sociale de façon à obtenir une meilleure couverture de presse.

2 Toutes les grandes religions ont ainsi compris depuis Plusieurs années l'importance de recourir aux communications pour affirmer leur existence et leur développement. Pour rejoindre des fidèles dispersés dans des adroits géographiques étendus, pour parler à des individus qui désertent les lieux du culte, les chefs des différentes religions ont choisi la voie et la voix des médias. Le pape convertit ses voyages à travers le monde en tribune Planétaire. L'ayatollah Khomeiny a bénéficié des médias Pour faire connaitre la fatwa contre Rushdie. Les télévangélistes américains ont abondamment utilisé la télévision comme nouveau cénacle.

De nombreuses études ont démontré que les grandes religions savent aujourd'hui utiliser de façon intelligente et efficace les différentes stratégies de communication, même si elles ont été lentes à le faire et même s'il existe toujours une certaine réserve à cet effet. Notre intervention se situe sur un autre plan. Plutôt que de se demander comment les églises utilisent les communications, nous allons inverser la proposition et nous demander comment les médias, comme institution sociale, ont imposé un nouveau discours à la 
religion. Nous allons illustrer ces propos en faisant plus souvent qu'autrement référence à l'Église catholique.

4 Notre objectif est de démontrer que les médias jouent dans les pays développés un rôle de premier plan dans la définition des enjeux sociaux, qu'ils exercent une certaine hégémonie sur nos façons de penser, d'être et de faire et que la religion n'échappe pas à leur emprise : elle s'y plie comme les autres instances sociales. Alors que la religion catholique, par exemple, imposait jadis ses règles du jeu dans la gestion du discours public - de façon musclée sous l'Inquisition, de façon directe avec les excommunications et l'index - ce sont maintenant les médias qui forcent la religion à se redéfinir sur la place publique, en amplifiant, par exemple, les contradictions dont elle est animée.

Altheide et le champ naguère investi par l'église. Et ce large champ est maintenant dominé par les médias. De ce fait, même la religion participe à la culture des médias et opère dans une large mesure à partir de la logique médiatique.

Il n'est pas de notre propos de nier l'importance de la famille, de l'école, du milieu, de l'église dans l'éveil et la socialisation à la religion. Il faut aussi reconnaitre qu'aujourd'hui, il existe des sectes religieuses et des segments de grandes religions (les cloitrés par exemple) qui se sont construits et ont vécu en marge des médias, soit de façon discrète ou secrète. Pour atteindre à la notoriété publique comme le recherchent toutes les grandes religions du monde, il faut nécessairement composer avec l'univers des médias. Et ceux qui pratiquent une certaine réserve publique et qui ouvrent quand même la porte aux médias, comme ces chœurs de moine qui ont accepté d'enregistrer du chant grégorien, sont aspirés contre leur gré dans le gouffre médiatique.

Ce serait une tautologie d'affirmer qu'il n'y a pas d'existence sociale sans la communication. Mais il fut un temps où tantôt le politique, tantôt le militaire, tantôt le religieux, tantôt l'économique, dictait les règles du jeu de cette existence. S'il est vrai que, dans un système social donné, toutes les institutions sont interreliées les unes aux autres, ceci n'exclut pas l'hégémonie que peut prendre l'une d'entre elles sur les autres. Altheide et Snow (1979, P. 199) rappellent qu'alors que la religion était l'institution dominante de son temps, elle présidait aux formes d'expression de cette époque : le calendrier, la danse, la mode, l'art étaient marqués du sceau de la religion, aujourd'hui, c'est le contenu de la télévision qui rythme les soirées et ce sont les émissions qui structurent les valeurs.

Le texte qui suit rappelle d'abord que les médias ont toujours constitué une menace pour l'Église catholique. Et qu'à défaut d'avoir pu les asservir malgré de nombreuses tentatives, incapables de les dominer, elle a appris à s'en servir avant de devoir s'y soumettre.

\section{L'émergence des communications dans la religion catholique}

9 L'Église catholique a toujours manifesté une position ambiguë face aux médias. Autant ceux-ci ont exercé un attrait certain sur elle, autant ils ont provoqué une crainte maladive de ses effets pervers, crainte qui a alimenté une forme de méfiance continuelle. 


\section{Un peu d'histoire}

10 Si l'on regarde depuis trois siècles la position de l'Église romaine face aux communications sociales, on se rend compte que cette communication civile venait directement remettre en cause le pouvoir de l'Église de dicter la façon de voir le monde.

11 Le siècle des Lumières, avec sa promotion des droits de l'homme et des idées de liberté et d'égalité entre les hommes, fut immédiatement perçu par l'Église comme une atteinte à son autorité et une menace pour les bonnes mœurs et la foi des peuples. Sa réaction ne se fit donc pas attendre longtemps et ce furent les moyens permettant la libre circulation de pensée (les livres et la presse) qui devinrent en premier lieu la cible des critiques ferventes.

«Avec la peste contagieuse des livres déversés sur le peuple chrétien, écrivit en 1766 le pape Clément XIII dans son encyclique Christianae reipublicae salus, des hommes égarés, attachés à des mensonges et éloignés de la sainte doctrine, troublent la pure source de la foi et battent en brèche les fondements de la religion (...). Il faut combattre résolument le fléau mortel de tant de livres » (Médiathec 1990, p. 18).

Un quart de siècle plus tard, Pie VI, dans sa lettre aux évêques de l'assemblée nationale de France condamnera de la même façon la liberté de presse qui accorde à l'individu : «le pouvoir de penser, de dire, d'écrire et même de faire imprimer impunément en matière de religion tout ce qui plaît » (Médiathec 1990, p. 18).

13 L'encyclique Mirari vos de Grégoire XVI, promulguée en 1832, poussera cette critique encore plus loin en parlant de " la liberté funeste, dont on ne put avoir assez d'horreurs » comme d'un danger réel non seulement pour l'intégrité de l'Église, mais aussi celle de l'État.

14 Mais c'est le fameux Syllabus errorum (catalogue des erreurs modernes) de Pie IX, joint à son encyclique Quanta cura (1864) qui établira finalement un fossé béant entre la doctrine de l'Église et le monde moderne. La liberté d'expression et de pensée y sera solennellement condamnée comme étant la source de la « corruption morale et spirituelle des peuples » (Médiathec 1990, p. 21).

15 Le fait même que l'Église s'attaque avec autant de ferveur contre l'idée de la libre expression et de la diffusion de l'information ne fait que confirmer la puissance et l'importance des nouveaux modes de la communication sociale. La première réaction de l'Église fut donc de considérer ce nouveau phénomène comme un ennemi qu'il fallait combattre à tout prix. Et pourtant, il s'agissait là d'un Processus irréversible dont le développement allait échapper à tout contrôle, soit-il civil ou religieux.

Le Concile Vatican II promulgua, en 1963, le Décret sur les moyens de communication sociale (Ínter Mirifica). De tous les documents conciliaires, c'est celui qui a rencontré la plus grande opposition des Pères du Concile. Le document se divise en deux parties dont la première, doctrinale rappelle l'enseignement de l'Église dans l'usage des médias et la seconde, pastorale, donne les indications pratiques pour rendre cet usage efficace au service de l'Église, Néanmoins, dans sa totalité, le document peut être considéré comme une grande mise en garde contre ce qu'il, solennellement, appelle «les admirables découvertes techniques ». L'enthousiasme s'y mêle constamment avec la méfiance. On voit l'Église se placer «en dehors du monde des communications sociales, comme une espèce de chien de garde qui doit protéger le public contre les méfaits des médias, tout en 
utilisant en même temps les ressources quand il s'agit d'un service direct à réclamer " (Ruszkowski 1968, p. 31).

17 En somme, le décret dénote une perception utilitariste et instrumentale de la réalité médiatique, mais il n'intègre Pas, à l'exception d'une courte mention à l'article 8 par exemple, l'utilisation des médias pour mieux façonner l'opinion publique. Les critiques du document soulignent enfin son retard par rapport à l'enseignement de Pie XII (1939-1958), qui dans ses nombreux énoncés « amorça une tendance vers l'information démocratique, qui devrait être considérée comme le réel tournant de l'enseignement de l'Église à l'égard de la démocratie » (Cousineau 1973, p. 19).

Élaborée par la Commission pontificale pour les moyens de communication sociale et approuvée par le Pape Paul VI, l'Instruction pastorale Communion et Progrès, paraît le 25 mai 1971. La majorité du texte est consacrée à l'exposition des principes moraux qui devraient animer les attitudes des usagers et l'action des professionnels, tout en précisant la façon dont l'Église peut les utiliser : « Les moyens de communication sociale présentent donc un triple intérêt pour le peuple de Dieu : ils aident l'Église à se révéler au monde moderne; ils favorisent le dialogue à l'intérieur de l'Église; ils apprennent à l'Église les mentalités et les attitudes de l'homme contemporain » (article 125).

Néanmoins, l'Église n'oublie pas de prononcer une mise en garde contre certains moyens de communication qui, selon leur style propre, peuvent fausser ou donner une résonance prématurée aux opinions nouvelles des théologiens. "L'Église réalise que le monde des médias, c'est bien plus qu'un instrument d'évangélisation, c'est aussi tout un monde à évangéliser » (Sokolowski 1994, p. 20).

20 L'instruction pastorale Aetatis Novae, de 1992, reconnaît que «le grand aréopage contemporain des médias a été plus ou moins négligé par l'Église jusqu’à maintenant » (article 20). Plus que jamais avant, l'Église voit dans les moyens de la communication sociale une chance extraordinaire pour pouvoir communiquer son message au monde entier devenu un "grand village». Mais d'autre part, elle ne cessera pas de parler des conséquences imprévisibles et néfastes de ces mêmes moyens. C'est ainsi que tout en considérant les médias de masse comme "des dons de Dieu» (article 22), l'Église les traitera de «demi-bienfaits» responsables du «sécularisme, du consumérisme, du matérialisme, de la déshumanisation, etc.. » (article 13).

21 Mais elle reconnaît le rôle essentiel des médias dans la formation de l'opinion publique : «La réalité correspond à Ce que les médias définissent comme tel : ce que les médias ne reconnaissent pas explicitement paraît insignifiant» (article 4). "Pour démontrer les enjeux d'une politique irresponsable dans le domaine de la communication sociale, le document va au-delà des critiques habituelles qui dénonçaient la superficialité de la culture médiatique, l'invasion de la violence et de la pornographie. Cette fois-ci, c'est la loi du marché qui règne dans le système des médias qui sera dénoncée (article 5). Et ce sont les annonceurs publicitaires, accusés d'« exercer une influence anormale sur le contenu des médias » qui se trouvent au centre de la critique » (Sokolowski 1994, p. 22).

En dénonçant les groupes de pression qui s'approprient la domination des médias, l'Église réclame "son Propre droit d'accès aux médias » car ce droit fait partie «du droit à la liberté religieuse, lequel ne devrait pas être limité à la seule liberté du culte » (article 15).

Pour le dire dans les mots même de Jean-Paul II : "De grandes possibilités sont aujourd'hui offertes à la communication sociale, dans laquelle l'église reconnaît le signe de l'œuvre créatrice et rédemptrice de Dieu, que l'homme doit continuer. Ces instruments 
peuvent donc devenir de Puissants canaux de transmission de l'Évangile, soit au niveau de la pré-évangélisation, soit à celui de l'approfondissement ultérieur de la foi, pour favoriser la promotion humaine et chrétienne de la jeunesse ».

\section{Entre la crainte et l'attrait}

24 Ce survol historique démontre qu'au fur et à mesure que se développent les techniques de communication de masse, au fur et à mesure que se confirme leur puissance sociale, l'Église, comme l'État d'ailleurs, va en même temps les craindre et ressentir le besoin de les utiliser, pour enfin se plier à la routine et à la logique journalistiques si elle veut profiter de leur puissance, même si elle dénonce en même temps, les effets négatifs de cette puissance.

L'Église qui a tout fait pour condamner les médias, doit maintenant se plier à leurs règles si elle veut continuer d'exister comme partenaire social. Comme un mal nécessaire, l'Église s'est peu à peu convaincue que l'avènement des médias de masse était incontournable comme outil de diffusion, mais dangereux comme foyer des nouvelles valeurs sociales.

26 Certains vont même jusqu'à attribuer la perte des valeurs morales et religieuses à la télévision. Des groupes américains luttent contre la télévision amorale. Les médias sont devenus l'ennemi de la religion. Pour Babin (1986, p. 97), « ... la crise qui secoue la foi traditionnelle a deux causes : l'ébranlement de toutes nos traditions culturelles, morales et religieuses par les technologies nouvelles et les médias. Le décalage entre la foi exprimée par les églises et les attentes d'un sentiment religieux surchauffé par le langage audiovisuel ». Tout comme autrefois le livre était source d'éloignement de la parole de Dieu, aujourd'hui la télévision est devenue le lieu des perditions. Selon un sondage paru dans le US News (AFP 1996), la télévision est la mère de tous les maux pour une majorité d'Américains, c'est elle qui favorise le déclin de la ferveur religieuse, qui éloigne les individus des vraies valeurs...

27 L'Église catholique est par ailleurs fascinée par le pouvoir des médias. Si ceux-ci peuvent faire tant de mal, peut-être peuvent-ils également faire autant de bien. Elle s'est dotée de multiples instruments de propagation de la foi : les Offices de communication sociale, les librairies, les maisons d'édition, la presse spécialisée et maintenant des stations de radio et de télévision.

Mais cette aventure dans les médias est une démarche en quelque sorte fermée. On crée des instruments de communication pour parler aux convaincus. Au lieu de s'adresser à la grande presse et au grand public, on crée des médias spécialisés qui s'adressent à ceux qui sont déjà attirés par la foi. C'est ce que Tremblay (1986, p. 100) veut illustrer lorsqu'il parle de l'Église comme d'une « institution qui s'est retranchée à son tour dans un univers devenu parallèle à la vie des gens ». Et il poursuit: "La société permet le feedback, le provoque même. Les citoyens ont acquis de nouveaux comportements et plus profondément de nouvelles attitudes. Il y a dissonance pour eux lorsque dans une des sphères de leur vie, le feedback n'est pas requis ni souhaité et que la communication est toujours unidirectionnelle » (Tremblay 1986, p. 162).

Dans le même sens, Flipo (1984, p. 215) examine " quelles sont les traductions concrètes " du passage « d'une religion fermée à une religion ouverte ». 

Offices de communication sociale s'ouvrent sur le grand public et les médias de masse. Au Québec, par exemple, cet Office fournit aux médias les cotes d'appréciation des films présentés à la télévision.

\section{L'Église s'est mal adaptée aux médias}

Il s'agit pourtant là de gestes timides. Certains chercheurs constatent plutôt que l'Église a mis du temps à se mettre à l'heure des médias. Pour Tremblay : l'Église connaît mal les médias, donc communique mal. Si elle est souvent apparue comme experte en communication de groupe, elle semble moins habile dans son utilisation des médias électroniques de communication (Tremblay 1986, p. 100). "Elle ne s'est pas vraiment adaptée à une nouvelle approche dictée par la nature même de ces médias et c'est Peutêtre qu'elle n'en a pas une bonne compréhension. Cette conjoncture la place d'emblée dans une situation de mauvaise utilisation de ces médias familiers à l'homme d'aujourd'hui " (Tremblay 1986,p.100). Et il poursuit: "Certains événements massmédiatisés pendant lesquels l'Église a démontré un savoir-faire exceptionnel peuvent laisser croire qu'il est dans ses habitudes de communiquer facilement par les médias. Cette impression ne résiste pas à une étude plus approfondie »(Tremblay 1986, p. II).

«Si les Offices nationaux de communication sociale s'en tirent assez bien dans leurs relations avec les médias, la situation est beaucoup plus précaire dans les diocèses où la vie de la communauté chrétienne ne trouve pratiquement pas d'écho dans les médias de masse » (Tremblay 1986, p. ii).

\section{Les médias boudent et couvrent mal l'Église}

Une des preuves que l'Église s'est mal adaptée aux médias, c'est que ceux-ci boudent et couvrent mal ses activités. Flipo (1984, p. 230) affirmait qu'« Un fait est certain, les médias, sauf la presse confessionnelle, ne sont pas particulièrement demandeurs de nouvelles de l'Église ; c'est donc à celle-ci de faire les premiers pas vers eux, à les séduire en quelque sorte ».

L'étude qu'a menée Tremblay en 1986 lui a permis d'affirmer qu'il existe une réelle « sous représentation de la vie religieuse dans (les) médias » (Tremblay, 1986, p. II).

Daiber (1987, p. 6) en était arrivé à la même conclusion : «Les médias maintiennent une certaine indépendance face à la religion institutionnelle et se présentent comme facteurs autonomes face à elle ».

En 1992, l'Office national des communications sociales du Québec effectuait à son tour une recherche sur le contenu religieux dans la presse écrite au Québec. On remarque que la quantité de sujets traités et l'espace qu'ils occupent ont diminué depuis les années 1970. Selon l'analyse, la religion occupe seulement $1 \%$ de l'espace rédactionnel dans les journaux québécois, soit presque le même espace que la science (Arteau 1992, p. 54). On remarque également que l'information religieuse médiatisée reflète presque uniquement l'Église institutionnelle, c'est-à-dire les conférences épiscopales et l'enseignement de l'Église (Arteau, p. 54). Les médias ignorent l'église au quotidien. Dart et Allen (1993) suggèrent qu'il y a un univers qui sépare les médias de la religion. Les premiers recherchent des faits, alors que la seconde s'élève au dessus du monde rationnel en quête d'une vérité plus complexe. 
Les mêmes auteurs constatent de plus que les compétences des journalistes en matière religieuse sont restreintes. Et, de ce fait, ils traduisent mal la réalité religieuse, ont parfois des préjugés négatifs ou hostiles. Ce qui amène les autorités religieuses à considérer que les médias ne s'intéressent qu'à la controverse. L'Église se voit aujourd'hui condamnée à ne faire partie de la couverture de presse que lorsqu'elle est impliquée dans une histoire de mœurs ou d'abus de pouvoir. "Dans le domaine religieux (...) les crises font de meilleurs titres que les réussites » (Souchon 1994).

Compte tenu que $90 \%$ des Américains affirment croire en Dieu, compte tenu que les médias ne reflètent pas adéquatement cette réalité, il n'y a qu'un pas à faire pour affirmer que les médias ont développé une attitude biaisée face à la religion (Dart et Allen 1993, p. 6).

38 En fait, selon les évêques catholiques américains, les médias vont rejeter de la sphère publique les questions de religion pour les confiner dans le cœur de l'homme, sinon dans l'intimité de son foyer (cité par Dart et Allen, 1993, p. 12). Sinon, la télévision ne va traiter que des aspects séculiers de la religion : l'avortement, le sexe avant le mariage, etc.

Cette prise de conscience de la faible attention des médias à son endroit va amener l'Église à adopter des stratégies pour répondre à la logique journalistique.

\section{La nécessité, pour l'Église, d'avoir recours aux médias}

Le fossé qui s'est créé entre la religion catholique et les médias et en particulier la télévision est accentué par la place prise dans ces mêmes médias par les télévangélistes où la dimension spectacle, le charisme des vedettes, l'emportent presque sur le contenu.

De ce fait, l'Église catholique va comprendre que si elle ne veut pas rester à l'écart de ces mouvements, elle doit s'y plier. Il se développe alors toute une approche incitant la religion à se rapprocher des médias, à les utiliser et même à moduler son comportement pour s'ajuster à la nouvelle réalité qui la confronte.

Avec l'arrivée des médias de masse, pour la première fois dans l'histoire, l'Église a le moyen de faire parvenir son message à tous les hommes de la terre, de tous les continents et de tous les niveaux culturels. "Elle peut enfin aller enseigner à toutes les nations » (Ruszkowski 1968, p. 20).

3 Déjà, Buntix (1971, p. 140) exhortait l'Église à faire appel à une technique qui a rendu bien des services à la vie industrielle, à savoir, les relations publiques. «Grâce à elles, on peut parvenir à une formation sérieuse de l'opinion publique. (...) L'Église ne sera plus alors pour beaucoup une idée abstraite qu'ils identifient à « l'autorité », mais ils finiront par comprendre que l'Église, c'est eux-mêmes ".

44 La présence ambiante des médias rejoint l'homme dans tout son être, de quoi faire envie à l'Église. Celle-ci se sensibilise au phénomène de la communication par les médias et s'aperçoit qu'elle peut avoir une relation plus directe avec l'homme qu'elle rejoint plus facilement en éliminant les distances (Dubuc 1971, 97sq.)

Pour Gabel (1971, p.343), l'Église doit vivre avec les communications: «... il faut qu'existent pour le dialogue et l'information des voies et des moyens plus spontanés, plus souples, ouverts à tout le monde : ce sont ceux de la communication sociale et tout particulièrement de la presse ». 
En 1973, le père Pierre Arrupe, alors supérieur général des Jésuites, initia une étude pilote de trois ans pour découvrir comment la Société de Jésus pouvait aider l'Église à acquérir la connaissance scientifique utile dans le champ des communications. A la suite de cette expérience, était créé le « Research Facilitator Unit for Social Communication » en 1976. C'est dans le cadre de ce centre de recherche que le père William Biernatzki (1978) publia une série de 40 questions sur le rôle de l'Église face aux médias.

Une de ces questions mettait en relief le fait que les groupes de pression de toute nature pouvaient attirer sans difficulté l'attention des médias et qu'il devait être possible d'avoir recours aux mêmes stratégies pour encourager la promotion de la justice et de la dignité humaine (Biernatzki 1978, p. 10).

Flipo (1984) va ainsi demander à l'Église de changer de comportement et d'ajuster ses valeurs à la réalité actuelle -en particulier en ce qui touche la sexualité - si elle veut offrir un visage attrayant aux chrétiens. Pour lui, « Dieu n'a peut-être pas besoin du marketing mais l'Église, si. Ses règles sont les mêmes quel que soit le type d'organisation, et les prélats ne doivent pas se considérer comme des gestionnaires à part » (p. 231).

Et il poursuit (Flipo p. 227) : «L'institution ecclésiale doit attirer, intégrer et motiver un public à qui il faut donner des raisons de pratiquer et militer ».

À la suite de ces nombreux appels à l'utilisation des médias, l'Église s'y est mise mais souvent de façon maladroite. «La première et la grande tentation des hommes d'Église, lorsqu'ils interviennent, est celle de l'utilisation purement instrumentale des médias. Souvent, dans l'Église, on dit qu'il faut utiliser les médias pour annoncer la Bonne Nouvelle, comme on se servirait d'un porte-voix pour être mieux entendu, et par plus de monde. Mais si on utilise les médias comme le surfer utilise la vague qui le porte, on risque d'être entraîné loin des objectifs de départ » (Souchon, 1994).

Sachant mal gérer l'espace public par l'intermédiaire des médias, l'Église catholique va commencer à subir de façon désagréable la logique implacable des médias.

2 «Dans cette perspective, on ne traitera guère de l'Église que lorsqu'on pourra le faire sous le signe d'un des aspects de la trilogie : Sexe - Argent - Pouvoir» (Souchon 1994). Défavorisée par le contexte social, l'Église catholique doit plus que jamais avoir recours aux techniques de relations publiques pour espérer profiter de la visibilité offerte par les médias.

Il n'est plus question seulement de faire connaître la bonne parole, de diffuser les décisions et orientations de l'Église par la voie des médias, il faut maintenant apprendre à gérer l'Église par les communications, et non plus seulement à subir et à réagir aux assauts des médias.

Pour Lesly 1991 (p. 632), « Le changement a toujours fait partie de la religion, mais c'est la vitesse à laquelle se font les changements dans notre génération qui devient le défi majeur pour les relations publiques au sein des organisations religieuses. »

De nos jours, ce que l'on entend par l'Église et par communion ne se transmet plus de père en fils, mais par initiation. Il faut donc que la religion se déplace du côté où les gens s'initient au monde, c'est-à-dire les médias. Et il ne s'agit plus seulement de déterminer l'impact que la «massmédiatisation du message évangélique peut avoir sur la véritable dimension des pratiques chrétiennes » (Tremblay 1986, p. 180), mais de participer à la définition de tous les enjeux sociaux par l'utilisation des médias.

«L'Église du XXI ${ }^{\mathrm{e}}$ siècle... ne pourra pas plus se passer des médias qu'elle ne pouvait se passer des cathédrales, de la musique et de l'art sacré aux siècles précédents. Et 
elle ne pourra pas non plus faire l'économie d'une réflexion sur ce qui est devenu le nerf de la société moderne : les médias eux-mêmes » (De Virieu, 1991 p. 33).

\section{Le pouvoir des médias}

56 D'où qu'on se retourne, toutes les réflexions exigent donc que l'Église se mette à l'heure des médias, apprenne à s'en servir, développe des stratégies adéquates. En fait, l'Église doit se subordonner aux médias pour rivaliser avec les autres instances sociales.

Il ne s'agit pas d'évaluer le vrai pouvoir des médias, ou ce qu'ils pourraient apporter à l'Église, car même si ceux-ci n'avaient aucune importance réelle dans la définition des enjeux, le pouvoir qu'on leur prête suffit pour qu'on doive en tenir compte. Le simple fait de passer 25 heures Par semaine devant la télévision pour un individu n'a Peut-être aucun effet sur lui, mais témoigne certes d'un mode de vie.

Désormais, l'Église va se définir en fonction de son savoir-faire dans l'utilisation des médias. Pour les uns, il s'agit d'un réel pouvoir à partager ; pour les autres, ce serait plutôt un outil puissant de diffusion. Mais dans un Cas comme dans l'autre, elle doit se plier à leur présence, à leur existence et à leurs façons de faire. Car qu'elle en soit agacée ou fascinée, l'Église catholique se définit aujourd'hui sur la place publique, dans et par les médias. De ce fait, elle subit le poids des médias dans sa façon d'être. Si chacun des déplacements du pape reçoit une couverture de presse internationale soigneusement prépa-fée, le moindre toussotement du pape subit le même traitement, de même que toutes les controverses suscitées par l'Église et ses apôtres.

La voix puissante du média comme son œil accusateur imposent désormais leur présence. Pour Olivenstein $(1988$, p. 10) : "On découvre également... que la presse peut être une arme extraordinaire pour obtenir du pouvoir, pour saisir du pouvoir et je crois que ça c'est une raison fondamentale que beaucoup de gens ont retenue... »

\section{La logique des médias a modifié la logique de l'église}

Il fut un temps, où lorsque les médias ne répondaient pas adéquatement aux vues de l'Église, les médias devaient plier l'échine (de Bonville 1988). L'Église n'a plus l'index, ni l'efficacité de l'excommunication pour imposer ses choix. Aujourd'hui, si l'Église ne s'ajuste pas aux médias, c'est elle qui plie l'échine. Car l'Église ne contrôle plus les règles du jeu qui prévalent dans la grande presse. Ces règles s'articulent autour de la notion de nouvelles. Un média doit vendre pour subsister. Et pour vendre, il doit attirer l'intérêt des lecteurs. Et pour ce faire, le média doit rechercher les écarts à la norme. Derrière chaque page de journal, chaque bulletin de nouvelles, il y a fortement dessinée une norme sociale dont on ne fait apparaître que les écarts. Ceux-ci se déploient entre le héros et le vilain pour reconstruire les nouvelles à la manière des contes russes de Propp (Brémond 1966).

Or aujourd'hui, le héros et le vilain sont séculiers. Que l'Église désire parler du spirituel, des valeurs fondamentales qui doivent guider l'être humain dans son développement, des valeurs spirituelles qui accompagnent le cheminement de chaque être, les médias veulent parler de héros, d'argent, de sexe, de controverses. C'est l'implication séculière de Mère Térésa auprès des affligés et non sa spiritualité qui a été dispersée par les médias. Ce sont les poussées sexuelles de certains membres de l'Église qui sont portées au voyeurisme public, et non l'âme profonde de ces mêmes êtres. Et lorsque le pape voyage, les médias 
traitent d'éléments relatifs à la parade, au spectacle et « font des efforts remarquables pour se mettre eux-mêmes en scène » (de Repentigny 1985, p. 32).

Les rôles sont inversés. L'Église qui châtiait les pénitents pour des pensées impures volontaires (Bechtel 1994), est maintenant châtiée pour des manquements aux vœux de célibat. Et l'Église ne peut plus se cacher. Car la logique des médias a modulé la hiérarchie des sources. Si un évêque officie un service religieux pour la journée des chauffeurs de taxi, il fera la nouvelle. Si un séminariste accuse un évêque d'abus sexuels, l'évêque sera toujours au centre de la nouvelle, mais en tant qu'accusé.

Cette logique a poussé certains auteurs comme Flipo (1984) à demander à l'Église de se redéfinir pour mieux réussir son marketing. Dans ce nouveau marketing social, "l'information qu'elle (la communication) véhicule sera le Produit lui-même » (De Guise 1991, p. 310). L'important, ce n'est plus l'Église, mais la façon de la faire connaître, aimer, apprécier. Pour Flipo (p. 231), « Du discours et de l'acte, c'est le dernier qui est retenu » dans les médias ".

Et l'Église a peu de choix dans cette lutte pour l'existence sociale. La nécessité de se plier à cette logique s'impose car à défaut de gérer cette logique, on en est réduit à la subir. Or nous avons vu dans le chapitre précédent les retards pris par l'église pour s'en rendre compte.

Voyons donc quelques-uns des effets de cette logique marchande des médias. Et les changements évidents apportés par la présence des médias.

\section{La force de l'autorité}

Les médias ont une prédilection pour les autorités (Gandy 1982) comme source première d'information. Et à ce titre, l'Église catholique a toujours su tirer son épingle du jeu. L'Église «a toujours su communiquer. Tant qu'elle est restée dans la civilisation de la parole proclamée ou celle du livre, elle réussissait à se faire entendre d'une façon satisfaisante » (Tremblay, 1986, p. 93).

67 L'Église catholique étant fortement hiérarchisée et presque militarisée, les ordres viennent d'en haut et on doit s'y soumettre. L'Église n'aime pas beaucoup qu'on remette en cause, sur la place publique, ses décisions. Le débat sur la position de l'église catholique française sur le condom en est un récent exemple (Baillargeon 1996). Peu importe ce que pense l'épiscopat français, c'est Rome qui décide ce qu'il faut penser du condom.

68 L'Église est fondamentalement une institution hiérarchique où le croyant est considéré comme un sujet et la hiérarchie comme un pouvoir. Pour la communication, nous sommes en présence d'une société autoritaire. La communication y est, la plupart du temps, initiée par une autorité et c'est cette communication que recherchent à l'évidence les médias. L'autre communication, celle qui vient de la vie de la communauté chrétienne, est marginale et traitée comme un fait divers. De ce fait, la voix de l'autorité aura un accès direct aux médias. Et le statut d'autorité s'acquiert par la légitimité dans un secteur d'activités. Lorsque cette légitimité est acquise, c'est à son représentant que revient d'office l'espace/temps médiatique. Les médias donnent d'abord la parole aux élites, aux vedettes, aux détenteurs de pouvoir. L'important n'est pas tant ce qu'ils disent que ce qu'ils représentent. Et de ce qu'ils disent, ce sont les éléments de controverse qui 
s'imposent dans le temps. C'est ainsi qu'un jour l'autorité religieuse décréta que la terre n'était pas ronde, ou que le condom n'était pas une solution au sida.

Dans son étude sur la couverture religieuse au Québec, Tremblay (1986, p. 22) en arriva à la conclusion suivante : « La constante la plus significative de cet échantillon d'une année dans la presse quotidienne québécoise est que la nouvelle religieuse s'articule surtout autour de l'Église institution, de la hiérarchie ».

70 Cette conclusion lui était confirmée par l'analyse de la couverture de la visite du pape en septembre 1984 au Québec. « ... il se dégage de tout cela que c'est la hiérarchie, le pouvoir, qui intéresse les médias. La structure Pyramidale où il n'y a que la parole d'en haut qui mérite attention et traitement journalistique, reste fermement ancrée dans l'esprit des « faiseurs » d'images et des reporters d'événements » (Tremblay 1986, p. 4).

71 Mais il rajoute que s'il est vrai que l'autorité attire l'attention des médias, ceux-ci n'en ont que pour l'événementiel et qu'aussitôt le héros disparu, cesse toute information profonde sur la religion. Et en cela, il rejoint la même conclusion avancée par Charron (1991) dans son étude sur les groupes contestataires. Ce que retiennent les médias, c'est davantage l'événement que le contenu de l'événement.

\section{La prise de la parole}

72 Le corollaire de cette logique médiatique, c'est donc, Pour toute autorité, de prendre la parole le plus souvent Possible et de s'affirmer sur tous les plans de l'actualité Pour occuper l'espace médiatique.

\section{S'affirmer}

73 Dès lors, dans la logique des médias, si l'autorité prend la parole, son statut justifie le discours qui s'ensuit. Alors, au lieu de se cantonner dans ses retranchements religieux, l'Église va se séculariser dans ses débats, pour obtenir une Plus grande visibilité sociale. «Les places se brouillent, celle des institutions comme celles des individus :... l'Église intervient de plus en plus dans le débat public alors qu'elle n'a plus prise sur les comportements privés » (Chambat et Ehrenberg 1993).

74 Prises de position sur la pauvreté, l'environnement, la famille, la langue, le partenariat social, l'ouverture des commerces le dimanche, la violence à la télévision, le salaire des riches, l'Église est de tous les débats. En novembre 1993, les médias laissaient savoir que l'Église anglicane d'Angleterre ne voyait pas d'un bon œil l'accession au trône de Charles, suite à sa conduite légère...

75 En paraphrasant Gritti (1970, p. 19), toutes ces déclarations « sont animées par un projet pastoral: mieux connaître les voies par lesquelles le message biblique atteint les mentalités, les langages, l'imaginaire, l'esprit et l'action des hommes d'aujourd'hui ».

76 L'Église a suivi, en ce sens, les habitudes des groupes de pression: intervenir aussi souvent que possible dans les médias sur des actions à mener, et non plus seulement sur une pensée à développer.

\section{Contester}

77 Fondamentalement, la logique des médias est construite autour de la confrontation des idées. Si le gouvernement propose une législation, l'opposition devra la battre en brèche. 
Si le patron adopte certaines mesures, le syndicat les contestera. À la gestation d'un groupe pro-choix (pour la libre disposition de son corps par la femme), naît un groupe pro-vie (anti-avortement).

Une des résultantes de cette logique médiatique, c'est que si une organisation ne participe pas directement à la prise de décision, la seule façon d'obtenir de l'espace/temps des médias, c'est de se définir, de se positionner par rapport aux élites décisionnelles. En d'autres mots, si dans un secteur d'activité, l'Église n'est plus considérée comme une instance décisionnelle pour la société, elle doit se positionner contre cette instance pour attirer l'attention des médias. En somme, l'autorité contestée demeure l'autorité et de ce fait mérite attention. Dès lors, la contestation articulée contre une forme d'autorité obtiendra nécessairement autant d'attention que l'autorité elle-même. Car si celle-ci exerce un attrait certain pour les médias, cet attrait est excité lorsque cette même autorité est objet de controverses. Et si la parole ne suffit pas pour attirer l'attention, une action spectaculaire ou originale fera l'affaire.

79 Mgr Jacques Gaillot, ex-évêque d'Evreux en Normandie, du fait de son autorité légitime a obtenu une renommée intéressante. Mais en plus, il a contesté l'autorité suprême, ce qui lui a valu une renommée encore plus grande. Mgr Lefebvre avait bénéficié auparavant du même traitement.

80 La logique des médias a toutefois son poids. C'est que cette renommée qui fait vendre les médias, qui procure momentanément gloire et attention, sème des effets secondaires : les nouveaux héros paient sur le plan individuel leur incartade face à leurs autorités.

81 Et à côté de ces faits d'armes de certains religieux qui recherchent l'attention des médias, il y a les médias qui recherchent chez les religieux des méfaits d'âme. La religion a subi des attaques comme la politique : abus sexuels, homosexualité, avortement, célibat des prêtres, femmes et religion, prédicateurs et leur débauche, malversations financières... L'autorité est donnée en pâture à la débauche médiatique au nom de la transparence, au risque d'affaiblir le sens des institutions mises en cause. La somme des erreurs de parcours de certains membres d'une organisation peut aujourd'hui jeter le discrédit sur cette organisation, alors que celle-ci constitue une institution en soi au-delà de tout soupçon. Cette logique médiatique va donc obliger les institutions à adopter des règles de comportement et à poser des gestes publics qui n'ont de véritables sens que dans une logique médiatique. Désormais, l'Église ne réagit plus seulement en fonction de ses principes, mais aussi en fonction de l'image que les médias projettent d'elle-même. Nous y reviendrons.

82 C'est ainsi qu'en Autriche, «l'archevêque de Vienne, Hans Hermann Groër, a annoncé sa démission... à la suite des accusations de pédophilie portées par d'anciens séminaristes. La hiérarchie catholique espère que cette décision, rendue publique lors de la grand-messe de l'Assomption à la cathédrale Saint-Etienne, ramènera le calme dans l'Église d'Autriche, en ébullition depuis l'affaire Groër » (Le Monde, 17 août 1995 p. 3).

83 Lorsque les médias ont commencé à traiter ouvertement de l'Église, celle-ci a perdu la protection dont elle avait toujours joui. Des frictions et des scandales jusqu'alors cachés étaient mis à jour. Rabaissée au niveau de toute autre organisation, sujette aux critiques, elle dut apprendre à travailler avec cette nouvelle réalité. L'Église est maintenant prisonnière d'une nouvelle logique: celle de devoir répondre aux attaques, de devoir s'excuser, de devoir accepter d'être poursuivie devant les tribunaux. L'Église a perdu le leadership du discours. Elle le subit. 


\section{Le culte de la vedette} conduite sexuelle rigide, les médias présenteront comme presque normale une certaine forme de débauche que pratiquent les artistes par exemple. Alors que la violence est bannie comme valeur sociale, les médias présenteront diverses formes de violence sans restriction. Il existe donc une culture des médias qui leur est spécifique. Lundby (1989) n'hésite pas à opposer et relier les notions de culture religieuse et de culture médiatique.

\section{La force de la télévision} l'activité qui accapare chaque semaine le plus grand nombre d'heures. Dans les multiples sondages réalisés dans les pays développés, la télévision est considérée comme la source d'information la Plus importante.

Comme élément de formation, il faut savoir qu'un enfant nord-américain de 10 ans par exemple a déjà passé plus de temps devant la télévision qu'à l'école. C'est ce qui amena Biernatzki $(1978$, p. 5) à constater le fait que la socialisation passe désormais autant par la télévision que par la famille. Dès lors, pour forcer l'acculturation aux valeurs religieuses, il fallait nécessairement se plier au mode d'appropriation de la réalité le plus suivi par les jeunes.

Communication et organisation, 9 | 2012 

Parler de l'Église à la télévision, c'est non seulement rejoindre des gens nouveaux, mais c'est aussi faire connaître l'Église à de nouvelles personnes. Mais la télévision, en privilégiant les symboles visuels autant qu'oraux, transpose le sens du message (Nidal 1972, p. 151).

\section{Le spectaculaire}

93 Il existe un certain consensus chez les chercheurs pour reconnaitre l'appétit sans borne des médias pour le spectaculaire. Certains condamneront cette tendance et d'autres tenteront de l'expliquer. Transposée dans le champ de la religion, cette approche aura comme effet de traduire toute information en divertissement. En décembre 1993, le Time consacrait sa page couverture et son article principal au phénomène des Anges. En avril 1995, le Time s'attaquait en première page au problème des miracles avec en sous-titre: «Ciel qu'est-ce qui se passe?». La religion est présentée sous des thématiques accrocheuses pour les lecteurs. Chaque année, on fait grand état de toute apparition de la vierge à l'un ou l'autre endroit du globe.

Tillard (1986, p. 528) ajoute que « dans les programmes chrétiens ou dans les émissions religieuses, on nous donne l'impression qu'on veut annoncer Jésus-Christ en le mettant comme en dehors de l'Église. De plus, les mass-media, en s'appuyant parfois lourdement sur les apparitions, les guérisons, les "miracles», déplacent le point d'appui de l'évangélisation... »

95 Devant le constat que tout est divertissement à la télévision et même les informations, Biernatzki (1978, p. 10) se demande s'il ne serait pas plus judicieux d'adopter le même format pour les programmes religieux.

96 J. Olfredo déclarait: "Comme toutes les institutions, l'Église est bien obligée de se montrer, et de prendre le risque de l'Église-spectacle, de même que l'on parlait naguère de l'Etat-spectacle » (cité dans Barthélémy 1985).

«Les manifestations religieuses prennent une dimension de plus en plus spectaculaires. Les visites du pape ressemblent maintenant à des shows : on y vient moins pour écouter un message du Saint-Père que pour le voir. Sans adhérer, on est séduit. La séduction... Un mot dangereux puisque la volonté de l'Église n'est pas de plaire mais d'affirmer sa vérité en dépit des modes » (de Virieu 1990, p. 152).

\section{La globalisation}

La globalisation de l'information impose de nouvelles approches. Le pape maintenant parle au monde entier lorsqu'il s'exprime à la télévision. «En ce sens, l'informateur religieux devrait donc avoir la préoccupation de chercher parmi tous les événements, tous les faits d'Église, ceux qui collent d'une manière plus directe aux préoccupations de nos contemporains. Au moment où tout prend une dimension mondiale, il importe souverainement que l'informateur religieux révèle à ses lecteurs les activités, l'espérance et les souffrances de l'Église à travers le monde entier » notait déjà Gabel en 1971 (p. 192).

Biernatzki en 1978 (p.l1) reprenait le même discours et se demandait si l'internationalisation de la télévision n'affecterait pas la réalité de l'Église.

« De fait il est devenu assez difficile, pour ne pas dire impossible, de maintenir un rigide esprit de clocher devant le flot d'informations sur les autres religions, rendu souvent spectaculaire par les conflits locaux et des prises de position qui sont

Communication et organisation, 9 | 2012 
retransmises quotidiennement depuis toutes les parties $\mathrm{du}$ monde. Paradoxalement, loin de dispenser les figures politiques et religieuses du besoin de partir de chez elles, les médias ont fait que les voyages sont devenus indispensables pour accomplir leur mission. Dans l'environnement électronique, la communication religieuse ne signifie plus écrire des choses aux gens, comme le font habituellement les papes avec leurs encycliques; elle signifie désormais faire sentir sa présence avec la plus totale immédiateté - pour ne pas dire intimité - et partager son propre corps dans cette nouvelle sorte de communion que permettent les ondes" (De Kerckhove 1990, p. 108).

99 L'auteur insiste pour dire que les médias électroniques créent une sorte d'aura technique qui étend la présence active au-delà des limites immédiates du corps présent à la télévision (p. 108).

\section{L'importance de l'image}

La culture médiatique exige que les partenaires sociaux développent une image forte (Dagenais 1991). Qu'est-ce qu'une image forte ? C'est celle qu'attribuent les médias aux organisations. Et pour avoir bonne image, il faut correspondre aux règles non écrites, mais pourtant réelles, des médias qui regardent les institutions et les individus avec un prisme bien filtrant. Ce sont les médias qui créent les images. Et sur ces images, les médias recréent de la réalité. Et sur cette réalité, les organisations tissent leur image. Finalement, l'image d'une organisation est celle que lui prêtent les médias.

Il faut alors comprendre que différents chercheurs se sont penchés sur cette question et se sont demandés si l'Église ne devait pas se soucier davantage de son image (Biernatzki 1978, p. 11).

102 Dans le cas de l'Église de France, Flipo (1984) insiste sur l'importance de modifier son image dans les médias. Car pour lui, une image négative chasse les fidèles. La nouvelle culture des médias s'articule autour de l'impression transmise autant sinon plus que du contenu véhiculé.

Pour Tremblay (1991, p. 95), gérer son image, oser parler, se montrer, faire de l'Église une institution ouverte implique une négociation avec le public qui peut avoir des conséquences importantes pour l'Église : «Il faut cependant bien se rendre compte que la gestion de son image dans les médias amène à négocier son image dans le public, qui dit médiatisation dit aussi compromis, ce sont les règles du jeu. On ne peut pas se prévaloir d'un statut spécial dans un univers pluraliste ».

104 En fait, pour être davantage présente dans les médias, l'Église doit donc adapter son discours et sa façon d'agir au format que les médias préfèrent, c'est-à-dire aux événements sortant de l'ordinaire et particulièrement intéressants pour le public.

On peut comprendre alors pourquoi les visites du pape constituent des événements importants en soi car elles englobent tous les éléments de la culture des médias: la télévision, le spectaculaire, le pouvoir, la globalisation et l'image. Nous avons là un exemple intéressant de la façon dont les médias moulent les institutions qui les entourent. Le pape Jean-Paul II domine bien l'utilisation des médias par sa personnalité charismatique. Mais ses voyages, ses gestes (prendre un enfant dans ses bras), sont dictés par la culture des médias. 


\section{L'Église opte pour une logique mass-médiatique}

Désormais, à défaut de faire partager sa foi, l'Église catholique va affirmer sa présence. «Ainsi la pratique régulière peut décliner alors que la voix de l'Église a des échos grandissants par rapport à la sphère publique » (Rémy 1990 p. 30).

\section{La prise de la parole}

107 En s'affichant sur la place publique, mettant ainsi à profit son rôle de leader, l'Église va suivre l'exemple des grandes institutions. Si elle veut occuper l'espace public, elle n'a qu'à prendre la parole, qu'à organiser des manifestations qui vont gérer l'agenda des médias. Car désormais, ceux-ci vont se laisser happer par ceux qui créent la nouvelle. Les médias ont imposé une règle implacable : si vous voulez exister, attirez notre attention selon nos canons.

De ce fait, l'Église va se plier à ces règles. La première, c'est de dominer le discours d'une part en devançant l'appétit des journalistes et à les alimentant régulièrement, d'autre part en se rendant accessible à toutes leurs questions : c'est la naissance des porte-parole officiels qui vont apprendre à occuper le devant de la scène aussi souvent que possible.
"Jean-Paul II estime que les médias sont la chaire moderne d'où la société
d'aujourd'hui reçoit ses grandes orientations morales et spirituelles. C'est pourquoi
il est essentiel que l'Église monte directement en chaire avec ses propres stations et programmes de télévision, sa propre voix au service de la vérité et de l'amour... L'Église doit exercer le ministère de la communication, tout comme elle doit exercer son ministère auprès de ceux qui font la communication " (Kwiatkowskaviatteau 1986, p. 61).

109 L'Église a donc besoin des médias de masse pour faire passer son message et de ce fait va se fier à des représentants dynamiques qui savent communiquer avec les médias. Or les médias se laissent charmer par des événements tout autant que par le discours.

\section{Les événements}

110 Pour de Virieu (1991, p. 33), « Même le calendrier scolaire n'est plus rythmé par les fêtes religieuses. C'est donc à l'Église de créer l'événement ». Pour Flipo (1984, p. 231), l'Église doit se «montrer», oser "parler», poser des gestes, des «actes», "vendre du témoignage ». " Pour faire parler de soi (et être entendu) dans les médias, il ne faut pas parler, mais agir d'une manière inattendue, provocatrice » (p. 229).

111 Les paroles sont insuffisantes, notamment en raison de la vague de désacralisation qui touche aussi la religion et qui emporte les valeurs intangibles dans les pays développés. Autrement dit, l'Église doit gérer une politique d'image, en tenant compte de l'évolution des sociétés, et de la manière dont elle est perçue dans celle-ci. Pour Flipo (1984, p. 231), l'Église doit créer d'autres « événements, faire en sorte que les médias présentent l'image que l'Église veut se forger ». 


\section{Les outils de communication}

112 L'Église va mettre sur pied de très nombreux outils de communication pour rejoindre le public: presse périodique, émissions de radio et de télévision, postes de radio et de télévision religieuses. Au niveau des productions elles-mêmes, l'Église catholique possède des maisons d'édition, des librairies, des agences de presse catholique. Elle n'hésite plus à avoir recours à la publicité. Elle produit des guides à l'intention des journalistes comme celui de l'agence de presse catholique des États-Unis, Catholic News Service, qui, en 1991, publiait sous le titre, Stylebook on Religion, A reference guide and usage manual, un ouvrage de 215 pages sous forme de dictionnaire. On y retrouve environ 1000 références destinées à faciliter le travail des journalistes dans le domaine religieux. Une annexe présente les différentes structures du Vatican et de la Conférence des évêques américains et donne les abréviations des ordres religieux masculins et féminins.

Enfin, l'Église n'hésitera plus à s'ouvrir sur l'inforoute électronique où non seulement elle pourra rejoindre les fidèles d'où qu'ils soient, mais recevoir d'eux un échange continu (Jean-Paul II, 1989).

\section{Les relations de presse}

Pour attirer l'attention des médias sur ces initiatives, l'Église va organiser autour d'elles d'autres manifestations. Celles-ci vont prendre toutes sortes de forme : des PRIX décernés aux meilleures publications, aux meilleures vidéos, aux meilleures initiatives religieuses, aux meilleures publicités; des RENCONTRES de diverses natures comme des séminaires, des colloques, des conférences, des congrès, des semaines thématiques; des MANIFESTATIONS ponctuelles comme les messes de bénédiction pour différentes circonstances : le début des vendanges, de la moisson. À tous ces événements, les médias seront conviés, recevront toute documentation pertinente et auront droit aux propos bien sentis des porte-parole (Dagenais 1996).

\section{Les groupes de pression}

Et pour multiplier ses voix dans les médias, l'Église va s'organiser en différents groupements que l'on peut assimiler aux groupes de pression et qui auront les mêmes tâches : faire entendre sur la place publique ses souhaits, revendications, contestations, supports ou condamnations de certaines mesures. De plus, ces groupes pourront exercer un lobby d'autant plus efficaces qu'il se présentera sous le signe de la multiplicité. Ce n'est plus l'Église catholique romaine qui parle mais l'une et l'autre des Associations nationales et internationales des périodiques catholiques, de la radio et de la télévision catholiques, des journalistes (L'UNION CATHOLIQUE INTERNATIONALE DE LA PRESSE), des communicateurs catholiques, des chrétiens œuvrant dans le domaine artistique. Ce seront des organismes de formation (INSTITUT INTERNATIONAL DE FORMATION EN COMMUNICATIONS SOCIALES ET RELIGIEUSES); des mouvements séculiers comme les regroupements catholiques d'agriculteurs, d'hommes d'affaires, etc.

116 Il apparait évident par les publications, les revues et les associations diverses que l'Église s'est construit un arsenal adéquat pour gérer ses relations avec les médias. Ce sont donc 
eux qui ont poussé la religion à s'investir dans ces champs d'activités. Dorénavant, la parole de l'Église s'articule en fonction des médias.

\section{Conclusion}

Vouloir résumer le comportement public d'une organisation par un seul paramètre, c'està-dire les médias, c'est méconnaître la complexité des organisations et du tissu social.

Mais ignorer la puissance des médias dans l'organisation tant de la pensée que du comportement de l'Église, c'est se priver d'un outil essentiel. Les télévangélistes américains ont compris très tôt le phénomène (Bruce, 1990) et ont su l'exploiter abondamment. Le Pape Jean-Paul II a bien senti la révolution qu'entraîne cet instrument aux mains de ceux qui savent s'y plier.

L'exemple de l'Église catholique, dans ses rapports avec la réalité, démontre qu'aujourd'hui la vie spirituelle d'une institution comme des individus ne peut plus être désincarnée. Elle doit se construire sur l'esprit du temps qu'a si bien décrit Edgar Morin, au début des années 60 .

AGENCE FRANCE-PRESSE 1996, "La télé, mère de tous les maux », Le Soleil de Québec, 9 avril, p. 13.

ALTHEIDE David L. et Robert P. Snow, 1979, Media Logic, Sage Library of Social Research 89, Beverly Hills, Sage publications, 256 p.

ARTEAU André 1992, «L'information religieuse dans les quotidiens », L'Église canadienne, vol 25, n² 2, pp. 53-56.

BABIN Pierre 1986, L'ère de la communication : réflexion chrétienne, Le Centurion-OCIC, Paris.

BAILLARGEON Stéphane 1996, "Les évêques de France et le préservatif: Pourquoi l'épiscopat français a viré capot en 24 heures ", Le Devoir, 19 février, p. 44.

BARTHELEMY 1985, «La scène catholique », Revue Autrement, n 75, décembre.

BIERNATZKI William E. 1978, Catholic communication research: topics and a rationale, The research Facilitator Unit for Social Communication, London, 36 p.

BREMOND Claude 1966, «La logique des possibles narratifs », Communications, Paris, Le Seuil, pp. 60-76.

BECHTEL Guy 1994, La chair, le diable et le confesseur, Paris, Plon, 437 p.

BUNTINX Hubert, 1971, L'église et le management, Editions Hommes et Techniques, Puteaux, $174 \mathrm{p}$.

BRUCE Steve 1990, Pray TV: Televangelism in America, London, New York, Routledge, 272 p. 104

CHAMBAT Pierre et Alain Ehrenberg 1993, «Les reality shows, nouvel âge télévisuel ?», Esprit, janvier, pp. 5 à 12.

CHARRON Jean 1991, «Les pseudo-événements de contestation : le cas du regroupement municipal autonome des jeunes ", (pp. 101-138) in Charron, Lemieux, Sauvageau (sous la direction de), Les journalistes, les médias et leurs sources, Boucherville (Québec), G. Morin, $237 \mathrm{p}$. 
COUSINEAU Jacques, 1973, Église et mass media: l'évolution des attitudes sur les communications sociales chez les hautes autorités de l'Église catholique, Office des communications sociales, $\mathrm{n}^{\circ} 16$, Montréal, octobre, $245 \mathrm{p}$.

DAGENAIS Bernard 1991, "Les institutions et la gestion de leur image publique », in Michel Beauchamp (sous la direction de) Communication publique et société, Montréal, Gaëtan Morin, pp. 337-369.

DAGENAIS Bernard 1996, La conférence de presse ou l'art de faire parler les autres, 1966, Québec, Presses de l'Université Laval, 242 p.

DAIBER Karl-Fritz 1987, « Avant propos : religion et médias populaires », Social Compass, vol. XXXIV, $n^{\circ} 1$, p. 4-9

DART J. et J. Allen 1993, Bridging the Gap : Religion and the newsmedia, The Freedom Forum, First Amendment center, Vanderbilt University.

de BONVILLE Jean 1988, La presse québécoise de 1884 à 1914: Genève d'un média de masse. Québec, Presses de l'Université Laval.

GUISE Jacques (de) 1991, «Le marketing social », in Beauchamp Michel (sous la direction de), Communication publique et société, Montréal, Gaëtan Morin, pp. 285-333.

de KERCKHOVE Derrick 1990, La civilisation vidéo-chrétienne, Paris, Éditions Retz/Atelier Alpha Bleue, $191 \mathrm{p}$.

de REPENTIGNY Michel 1985, «La visite du pape au Québec : spectacle et spiritualité », Communication, Editions Saint-Martin, pp. 32-43.

de VIRIEU François-Henri 1990, La médiacratie, Paris Flammarion, 1990.

de VIRIEU François-Henri 1991, "Dans l'ère de la médiacratie ", L'actualité religieuse dans le Monde, $\mathrm{n}^{\circ}$ 91, 15 juillet-août, pp. 31-33.

DUBUC Jean-Guy 1971, Mass media : pour ou contre Dieu, Beauchemin, Montréal, 119 p.

FLIPO Jean-Paul 1984, Le marketing de l'église, Editions du Cerf, Paris, 284 p.

GABEL Emile 1971, L'enjeu des médias, Marne, Paris, 469 p.

GANDY Oscar 1982, Beyond Agenda Setting: Information subsidies and public policy, Norfolk: Abex Publishing Co, $243 \mathrm{p}$.

GRITTI Jules 1970, Bible et techniques de masse, Paris, Editions Fleurus, 193 p.

JEAN-PAUL II 1989, "The Church Must Learn to Cope with Computer Culture », World Communications Day, 27 mai.

KWIATKOWSKAVIATTEAU Alexandra 1986, «Le pape communique... », Revue Médiapouvoirs, Paris, $\mathrm{n}^{\circ} 4$, septembre.

LESLY Philip 1991, Lesly's handbook of Public Relations and Communications, 4th Édition, Éditeur Philip Lesly.

LUNDBY Knut 1989 "Religion and the Media in the Nordic countries", Social compass, vol. $\mathrm{n}^{\circ} 37$, pp. 179-186.

MEDIATECH 1990, Les médias: textes des Églises, documents réunis et présentés par le groupe Médiatech de la Faculté de théologie de Lyon, Paris Centurion, $463 \mathrm{p}$.

NIDA Eugene A. 1972, Message and Mission : the communication of the Christian Faith, William Carey Library, South Pasadena. 


\section{RÉSUMÉS}

Pendant des siècles, l'Église catholique a imposé son emprise sur le monde séculier qu'elle dominait. Le calendrier, le rythme des saisons, la pensée, tout était dicté par l'Église. À l'aube du XXI ${ }^{\mathrm{e}}$ siècle, l'Église a subi l'hégémonie des médias, transformés en une véritable puissance en soi. D’abord craints puis condamnés par l'Église, les médias sont devenus au fil des ans des alliés indispensables. Aujourd'hui, ils s'affirment comme les maîtres auxquels doit se soumettre toute institution et ont réussi à imposer la logique de l'existence sociale. Paraître est aujourd'hui autant, sinon plus important, qu'être.

For centuries, the Catholic Church exerted its power on the secular world which she ruled: the calendar, cadence of the seasons, reasoning, everything was dictated by the Church. At the dawn of the XXI ${ }^{\text {st }}$ century, the Church has felt the supremacy of the media, becoming itself a real power. At first feared, then later condemned throughout the years, media has become an essential ally. Today, the media asserts itself as the leader to whom every institution has to bow to, and has succeeded in implanting the logic of social existence: the image is as important today, if not more so than the reality.

\section{AUTEUR}

\section{BERNARD DAGENAIS}

Bernard Dagenais est professeur au Département d'information et de communication de l'Université Laval. Il est l'auteur de nombreux articles et ouvrages sur la communication. 
Avant d'enseigner à l'université, il a pratiqué le métier de communicateur pendant 20 ans. 